ARTICLE

https://doi.org/10.1038/s41467-019-12068-x

\title{
Biomechanical signaling within the developing zebrafish heart attunes endocardial growth to myocardial chamber dimensions
}

\author{
Dorothee Bornhorst (1) ${ }^{1,2}$, Peng Xia (1) ${ }^{3}$, Hiroyuki Nakajima (1) 4, Chaitanya Dingare (iD) ${ }^{5}$, Wiebke Herzog (iD ${ }^{6,7}$, \\ Virginie Lecaudey (10 ${ }^{5}$, Naoki Mochizuki (1) ${ }^{4,8}$, Carl-Philipp Heisenberg (D) ${ }^{3}$, Deborah Yelon ${ }^{9}$ \& \\ Salim Abdelilah-Seyfried (D) ${ }^{1,2}$
}

Intra-organ communication guides morphogenetic processes that are essential for an organ to carry out complex physiological functions. In the heart, the growth of the myocardium is tightly coupled to that of the endocardium, a specialized endothelial tissue that lines its interior. Several molecular pathways have been implicated in the communication between these tissues including secreted factors, components of the extracellular matrix, or proteins involved in cell-cell communication. Yet, it is unknown how the growth of the endocardium is coordinated with that of the myocardium. Here, we show that an increased expansion of the myocardial atrial chamber volume generates higher junctional forces within endocardial cells. This leads to biomechanical signaling involving VE-cadherin, triggering nuclear localization of the Hippo pathway transcriptional regulator Yap1 and endocardial proliferation. Our work suggests that the growth of the endocardium results from myocardial chamber volume expansion and ends when the tension on the tissue is relaxed.

\footnotetext{
${ }^{1}$ Institute of Biochemistry and Biology, Potsdam University, D-14476 Potsdam, Germany. ${ }^{2}$ Institute of Molecular Biology, Hannover Medical School, D30625 Hannover, Germany. ${ }^{3}$ Institute of Science and Technology Austria, 3400 Klosterneuburg, Austria. ${ }^{4}$ Department of Cell Biology, National Cerebral and Cardiovascular Center Research Institute, Osaka 565-8565, Japan. ${ }^{5}$ Institute of Cell Biology and Neuroscience, Department of Developmental Biology of Vertebrates, Goethe Universität Frankfurt am Main, 60438 Frankfurt am Main, Germany. ${ }^{6}$ Max Planck Institute for Molecular Biomedicine, 48149 Münster, Germany. ${ }^{7}$ Cells-in-Motion Cluster of Excellence (EXC 1003-CiM), University of Münster, 48149 Münster, Germany. ${ }^{8}$ AMED-CREST. National Cerebral and Cardiovascular Center, Osaka 565-8565, Japan. ${ }^{9}$ Division of Biological Sciences, University of California, San Diego, La Jolla, CA 92093, USA.

Correspondence and requests for materials should be addressed to S.A.-S. (email: salim.seyfried@uni-potsdam.de)
} 
T he growth and morphogenesis of organs requires some means of intra-organ communication between its different tissues. In the heart, biochemical signaling of secreted factors such as Wnts, BMPs, FGFs, or components of the extracellular matrix and cell-cell communication via the Notch, Neuregulin/ErbB2, or Ephrin signaling pathways ${ }^{1-3}$ are involved. Yet, it is unknown which modes of communication coordinate the growth of the endocardium with that of the myocardium. In zebrafish, during the process of cardiac ballooning at 30-54 hours post fertilization (hpf), endocardial chambers grow by proliferation with neither accretion of cells from external sources, nor from cellular intermingling over the chamber boundary at the atrioventricular canal ${ }^{4}$. In striking contrast, the myocardium grows mostly through an accretion of cells to the chamber poles $^{5-8}$ and due to cell size increases ${ }^{9,10}$. Here, we use two different genetic conditions (loss of Nkx2.5 or increase in Wnt8a expression) that cause an exaggerated expansion of myocardial atrial chamber dimensions. Comparing these two extreme conditions to WT reveals that an increased expansion of myocardial atrial chamber dimensions is compensated by increased endocardial proliferation and cell numbers. We find that an increased expansion of the atrial chamber volume generates higher junctional forces within endocardial cell junctions which are transduced by the endothelial-specific adherens junction protein Cadherin-5 (VE-cadherin) to activate Hippo pathway transcriptional regulator Yap1-dependent endocardial proliferation.

\section{Results}

Endocardial cell proliferation increases in larger atrial chambers. To examine whether changes in the dimensions of the myocardial chambers affect endocardial cell numbers, we analyzed two well-established genetic conditions that cause changes in their relative dimensions. Upon heat shock at $24 \mathrm{hpf}$, the atrial myocardium of the zebrafish transgenic line $\mathrm{Tg}$ (hsp70l:wnt8a-

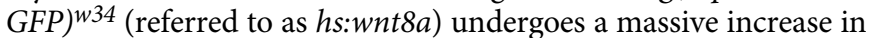
volume and cell numbers as determined at $52 \mathrm{hpf}$, whereas the ventricular chamber decreases in size $\mathrm{e}^{11}$. We also employed the $n k \times 2.5^{\text {vu179 }}$ mutant, which causes a comparable myocardial phenotype $^{12}$. In both genetic conditions, atrial chamber growth involves increases in myocardial cell numbers while numbers of ventricular cardiomyocytes are reduced (Supplementary Fig. 1) $11,13,14$. We reasoned that these two different genetic conditions would be good tools to study the response of the endocardium to this mode of myocardial atrial chamber expansion.

We found that under these two genetic conditions, the dimensions of the endocardial chambers underwent comparable changes at $52 \mathrm{hpf}$, which is the stage when relative chamber dimensions are established. Compared with WT, the overexpression of Wnt8a resulted in a relative shift of endocardial chamber dimensions with an enlarged atrium containing higher endocardial cell numbers and a smaller ventricle (Fig. 1a, b, g). To clearly identify chamber identities, the transgenic reporter line $\mathrm{Tg}$ $(f l t 1: Y F P)^{h u 4624}$ which mainly marks ventricular endocardial cells $^{15}$ was counter-stained with the myocardial atrial marker anti-Myosin heavy chain 6 (Myh6) which includes cells at the atrioventricular canal where Myh6-positive cardiomyocytes form a sharp boundary (Fig. 1d, e). Similarly, relative shifts in chamber dimensions with an increase of atrial endocardial cell numbers occurred in $n k x 2.5^{\text {vul79 }}$ mutants (Fig. 1c, g) ${ }^{13,14}$ which are phenotypically similar to the antisense oligonucleotide morpholino (MO)-mediated knock down of $\mathrm{Nkx} 2.5 / \mathrm{Nkx} 2.7^{13}$. The latter condition resulted in a smaller endocardial ventricular chamber, as indicated by the expression of $\mathrm{Tg}(f l t 1: Y F P)^{h u 4624}$ (Fig. 1f). These endocardial-specific changes mirrored those that occur
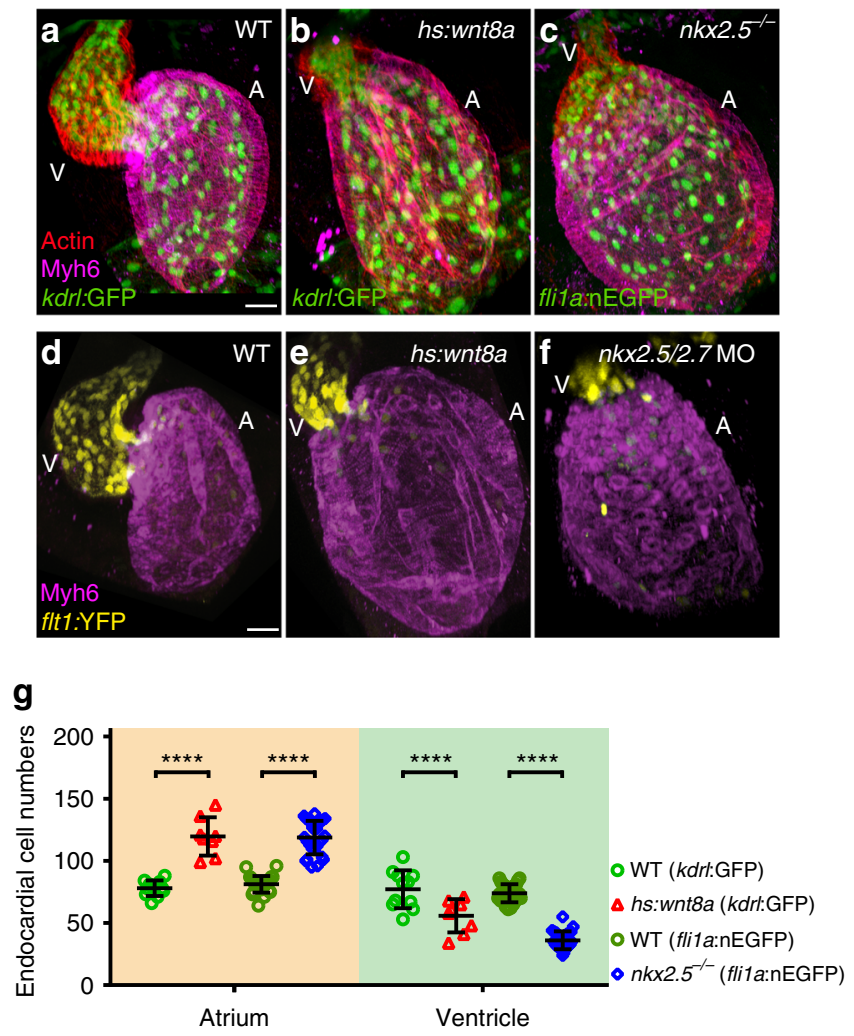

Fig. 1 Overexpression of Wnt8a or loss of $\mathrm{Nkx2.5}$ causes a shift in endocardial chamber proportions. a-c Reconstruction of confocal z-stacks show the endothelial-specific transgenic reporter lines $T g(k d r l: E G F P)^{\text {s } 843}$ or Tg(fli1a:nEGFP)y7 (green), Phalloidin 568-stained Actin (red) and anti-Myh6 labeling of the myocardial atrial chamber (magenta). Compared to (a) WT (b) overexpression of Wnt8a, or (c) the $n k x 2.5^{v u 179}$ mutation, causes a relative shift of endocardial chamber dimensions. d-f Reconstructions of confocal z-stacks shows that the arterial endothelial transgene $T g$ (flt 1:YFP) hu4624 marks the ventricular chamber whereas the myocardial atrial marker anti-Myh6 labels the atrial chamber. Compared to (d) WT, (e)

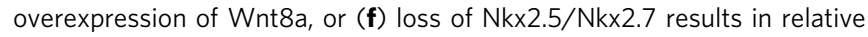
shifts of chamber dimensions as indicated by the ventricular-specific expression of $\mathrm{Tg}$ (flt1:YFP) ${ }^{\text {hu4624 }}$ within the endocardium. A, atrium; $\mathrm{V}$, ventricle. Scale bars, $30 \mu \mathrm{m}$. $\mathbf{g}$ Quantifications of endocardial cell numbers in WT ( $k d r l: G F P ~ n=11$ hearts, fli1a:nEGFP $n=27$ hearts), $T g$ (hsp70l:wnt8aGFP)w34 ( $n=8$ hearts), or $n k \times 2.5^{\text {vu179 }}$ mutants ( $n=27$ hearts) reveal that atrial endocardial cell numbers are significantly increased and ventricular endocardial cell numbers are significantly reduced compared to WT. Mean values \pm SD are shown. Two-way ANOVA was used to compare each condition with its WT control in each individual chamber $\left({ }^{\star \star \star \star} p<0.0001\right)$

within the myocardium under these conditions (Supplementary Fig. 1) $)^{11-13}$.

One explanation for the increased number of endocardial cells might be their proliferation. We found that the numbers of atrial and ventricular endocardial cells were identical between all genetic conditions analyzed at $30 \mathrm{hpf}$ (Fig. 2a). This indicates that the sizes of endocardial progenitor cell pools were not altered. Hence the respective proliferation rates were examined by injections of EdU into the circulatory system at $30 \mathrm{hpf}$ and analyzed its incorporation within endocardial cells at $52 \mathrm{hpf}$ (Fig. 2b). As a result, the rate of endocardial cell proliferation increased significantly within the atrium but not the ventricle compared to WT (Fig. 2c-f, s) upon Wnt8a overexpression (Fig. $2 \mathrm{~g}-\mathrm{j}, \mathrm{s}$ ), in $n k \times 2.5^{\text {vul } 179}$ mutants (Fig. $2 \mathrm{k}-\mathrm{n}, \mathrm{s}$ ), or upon knock down of $\mathrm{Nkx} 2.5 / \mathrm{Nkx} 2.7$ (Fig. 2o-r, s). In contrast, no increase in 


\section{a}
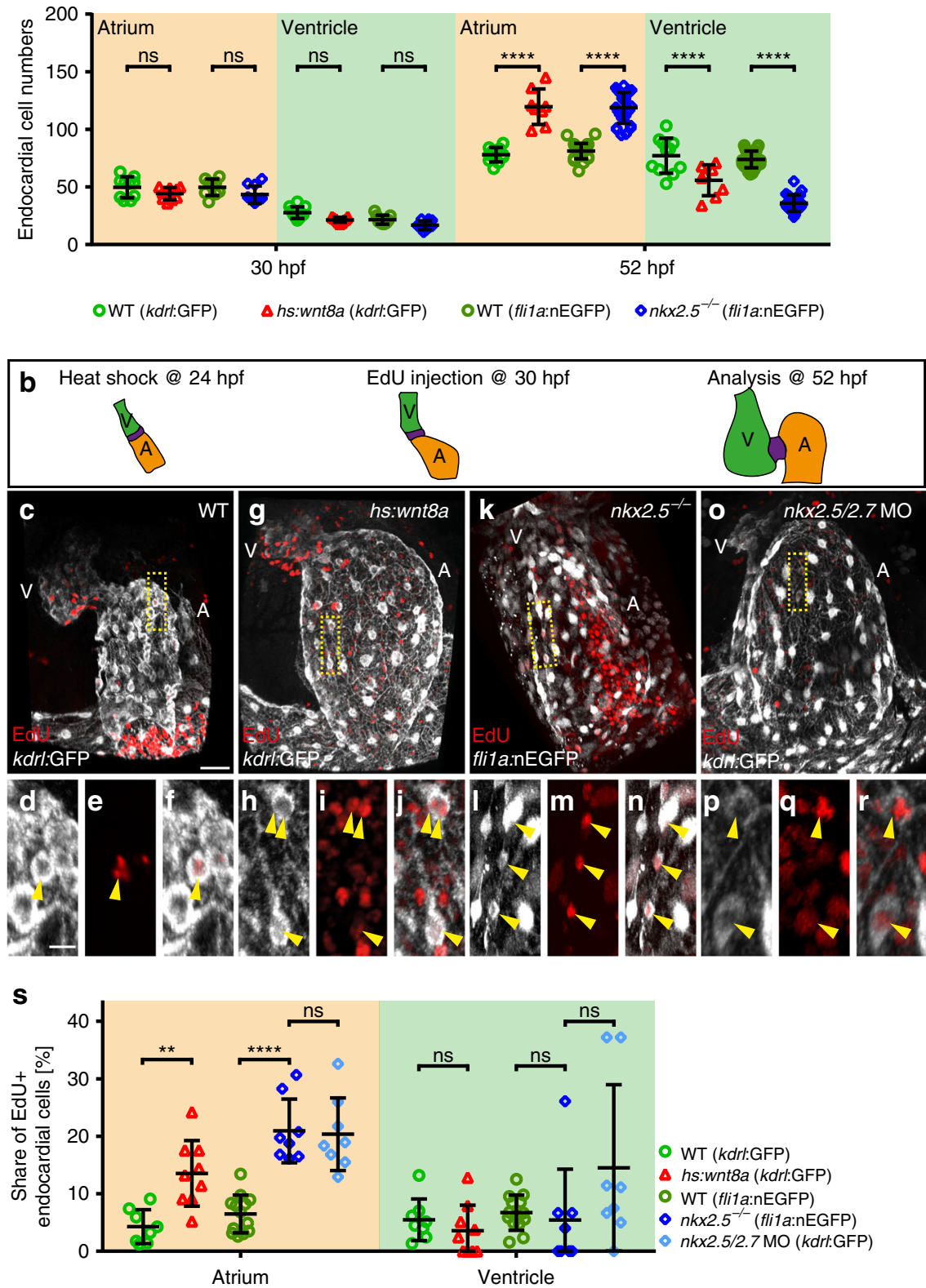

Fig. 2 Atrial endocardial cell proliferation is increased upon Wnt8a overexpression or in $n k \times 2.5^{v 4179}$ mutants. a Quantifications of endocardial cell numbers at $30 \mathrm{hpf}$ reveals that there is no increase of atrial endocardial cells in Tg(hsp70l:wnt8a-GFP)w34 ( $n=9$ hearts), or nkx2.5v179 mutant embryos ( $n=10$ hearts) compared with WT ( $k d r l: G F P ~ n=10$ hearts, flita:nEGFP $n=9$ hearts) at this stage. At the later heart ballooning stage (52 hpf), a significant increase of endocardial atrial cell numbers has occurred upon Wnt8a overexpression [Tg(hsp70l:wnt8a-GFP)w34 ( $n=8$ hearts) or in $n k \times 2.5^{\text {vul79 }}$ mutants $(n=27$ hearts) compared to WT ( $k d r l: G F P n=11$ hearts, flita:nEGFP $n=27$ hearts). Mean values \pm SD are shown. Two-way ANOVA was used to compare each condition with its WT control in each individual chamber (ns: not significant, ${ }^{\star \star \star} p<0.0001$ ). b Schematic model of the EdU assay used. WT and Tg(hsp70l: wnt8a-GFP)w34 transgenic embryos were heat shocked at $24 \mathrm{hpf}$. Embryos of all genotypes were injected with EdU into the circulatory system at 30 hpf and analyzed at $52 \mathrm{hpf}$. c, g, k, o Reconstructions of confocal z-stacks showing representative hearts at $52 \mathrm{hpf}$ of (c) WT, (g) upon Wnt8a overexpression, (k) in $n k \times 2.5^{\text {vu179 }}$ mutants, or (o) in $n k \times 2.5 / n k \times 2.7$ double morphants. Endocardial tissue is marked by $T g(k d r l: E G F P)$ s843 or Tg(fli7a:nEGFP)y7 reporters (white) and proliferative cells are marked by EdU incorporation (red). A, atrium; V, ventricle. Scale bars, $30 \mu \mathrm{m}$. $\mathbf{d}-\mathbf{f}, \mathbf{h}-\mathbf{j}$, I-n, p-r Shown are magnifications of insets (yellow boxes) with kdrl:GFP or fli7a:nEGFP/EdU double positive cells (yellow arrowhead). Single channel with kdrl:GFP or fli7a:nEGFP (d, $\mathbf{h}, \mathbf{I}$, $\mathbf{p}$ ), EdU incorporation $(\mathbf{e}, \mathbf{i}, \mathbf{m}, \mathbf{q})$ and the merge of both channels $(\mathbf{f}, \mathbf{j}, \mathbf{n}, \mathbf{r})$. Scale bars, $10 \mu \mathrm{m}$. $\mathbf{s}$ Quantifications of the share of EdU + atrial or ventricular endocardial cells relative to the total number of endocardial cells within the respective cardiac chamber in WT ( $k d r l: G F P n=8$ hearts, fli7a:nEGFP $n=13$ hearts), upon Wnt8a overexpression ( $n=9$ hearts), $n k \times 2.5^{v u 179}$ mutants ( $n=8$ hearts), or $n k \times 2.5 / n k x 2.7$ double morphants ( $n=8$ hearts). Upon Wnt8a

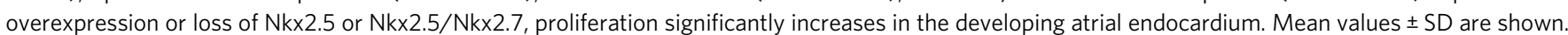
Two-way ANOVA was used to compare each condition with its WT control in each individual chamber (ns: not significant; ${ }^{\star \star \star} p<0.001,{ }^{\star \star \star \star} p<0.0001$ ) 
cell proliferation was observed upon Wnt8a overexpression within the myocardium despite strong EdU-labeling within some extra-cardiac tissues (Supplementary Fig. 2). This is similar to what has been reported for $n k x 2.5^{v u 179}$ mutants or $n k x 2.5 / n k x 2.7$ double morphants ${ }^{12,13}$. Hence, the increases in atrial endocardial cell numbers correspond with more proliferation.

Endocardial cell sizes are unaltered in enlarged atrial chambers. Using an in vivo multi-color mosaic labeling technique ${ }^{16}$ and morphometric measurements of immunolabeled hearts, we discovered that increased Wnt8a expression or loss of $\mathrm{Nkx} 2.5$ caused striking increases in the size of atrial cells within the myocardium (Supplementary Fig. 3 a-f; Supplementary Movies $1,2)$. We also used an alternative measurement and determined inter-nuclear distances in WT and upon Wnt8a overexpression, which provided the same result (Supplementary Fig. $3 g$ ). We wondered whether endocardial cell sizes were equally affected by the strong expansion of cardiac chamber dimensions. To quantify endocardial cell sizes, we measured at least 20 endocardial internuclear distances per heart using the endocardial marker lines $\mathrm{Tg}$ $(k d r l: E G F P)^{s 843}$ or $\mathrm{Tg}(\text { flila:nEGFP })^{y 7}$ at $52 \mathrm{hpf}$. Although atrial endocardial chambers were expanded under these conditions, Wnt8a overexpression (Fig. 3b, e-g) or loss of Nkx2.5 (Fig. 3d-g) did not result in significant changes in inter-nuclear distances or endocardial cell densities within the atrial endocardium compared to WT (Fig. 3a, c, e-g). Thus cell size increases are not responsible for the expansion of the atrial endocardial chamber in these two conditions.

Endocardial cell junctional tension increases within a larger atrium. Our observations suggested that myocardial chamber dimensions influence the proliferation of endocardial cells. This hinted at some form of intra-organ communication between the myocardium and endocardium. Cells within the endocardium and myocardium are separated by a layer of extracellular matrix (cardiac jelly) $^{2}$ which can propagate mechanical tension ${ }^{17,18}$. We hypothesized that when the size of endocardial cells remains constant, tensile forces on their cell junctions rise through the expansion of atrial chambers that occurs upon Wnt8a overexpression or loss of $\mathrm{Nkx} 2.5 / \mathrm{Nkx} 2.7$.

To address this issue, we adapted an ultraviolet (UV)-laser dissection set-up ${ }^{19}$ to measure cell junctional forces within the zebrafish endocardium. The transgenic reporter line $\operatorname{Tg}$ (act2: myl12.1-EGFP)e2212, which labels the non-muscle myosin II cytoskeleton, distinctly marks the sub-membranous compartment of endocardial cells ${ }^{20}$ which is distinct from the sarcomeric myocardium (Supplementary Movie 3). This permitted us to direct the UV-laser at cell junctions to measure the recoil of endocardial cell junctions upon dissection. To assess whether cell junction tensile forces differed between blood flow-parallel and cell junctions with an orientation perpendicular to the direction of blood flow, we performed measurements of recoil velocities in WT. This revealed no significant differences in initial recoil velocities between these different membrane compartments (Supplementary Fig. 4). To compare cell junctional forces, we targeted only the shorter perpendicular cell junction compartments of endocardial cells (red membrane compartments in Fig. $4 \mathrm{a}, \mathrm{b}$ ) and performed only one dissection per endocardium within a cell positioned near the inflow tract region to avoid tissue-relaxation upon neighboring cells (Fig. 4a, b).

To assess junctional tensile forces while the chamber expansion process was still ongoing at $40 \mathrm{hpf}$, we anaesthetized embryos and paused their heart beat with tricaine, which does not affect

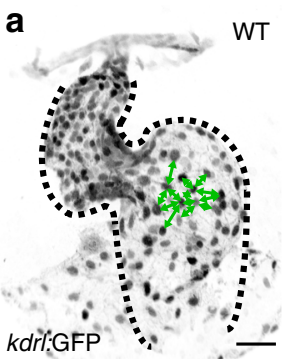

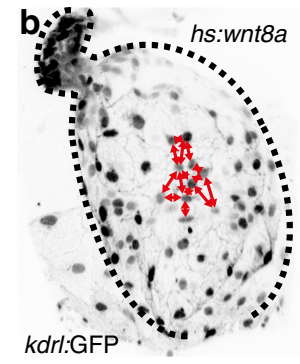

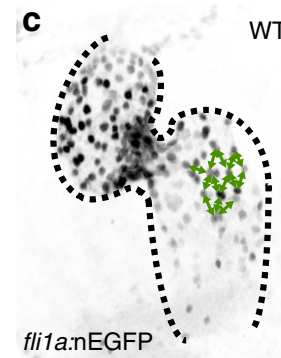

WT

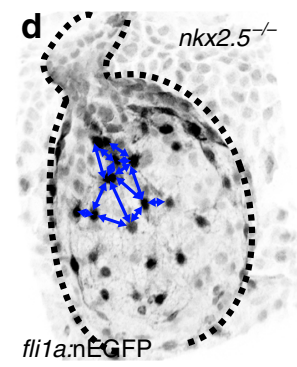

e

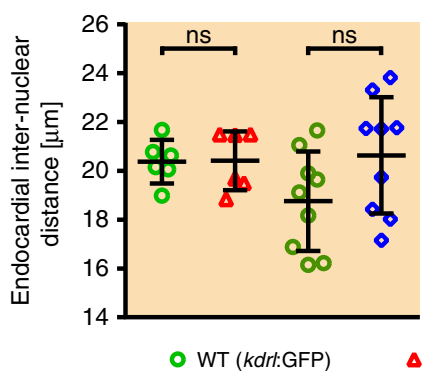

$\mathbf{f}$

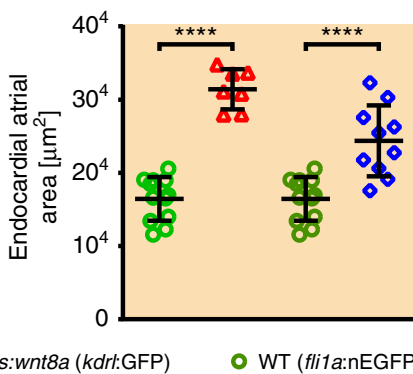

g

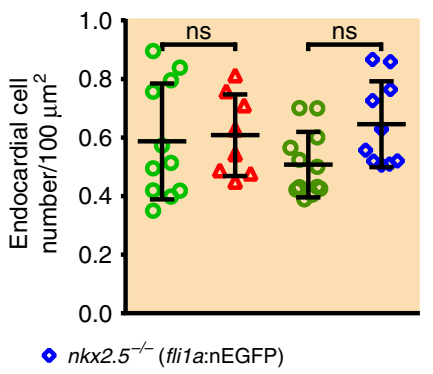

Fig. 3 Endocardial cell sizes are not altered upon atrial chamber expansion. a-d Reconstructions of confocal z-stacks of representative hearts at $52 \mathrm{hpf}$ of (a) WT, (b) upon Wnt8a overexpression, (c) WT, or (d) in $n k x 2.5^{\text {vul79 }}$ mutants. Endocardial GFP expression of $T g(k d r l: E G F P) s 843$ or $T g(f l i 7 a: n E G F P) y 7$ is inverted in black/white. Endocardial inter-nuclear distances are indicated by arrows and a dotted line indicates the outline of the endocardium. Scale bars, $30 \mu \mathrm{m}$. e Quantifications of endocardial inter-nuclear distances reveal no significant differences between WT ( $k d r l: G F P ~ n=6$ hearts, fli7a:nEGFP $n=9$ hearts), upon Wnt8a overexpression ( $n=6$ hearts), or in $n k x 2.5^{v u 179}$ mutants ( $n=9$ hearts). Each dot represents one heart with an average of at least 20 length measurements within the endocardial atrium. $\mathbf{f}$ Quantifications of the atrial endocardial surface area reveals a significant increase upon Wnt8a overexpression ( $n=7$ hearts) or loss of Nkx2.5 ( $n=10$ hearts) compared to WT hearts (kdrl:GFP $n=12$ hearts, flita:nEGFP $n=12$ hearts).

g Quantifications of the ratio of endocardial cell numbers relative to total atrial chamber area indicates no significant differences between WT ( $k d r l: G F P$

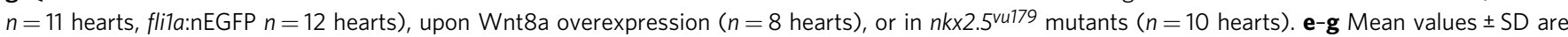
shown. One-way ANOVA was used to compare each condition with its WT control (ns: not significant, ${ }^{\star \star \star *} p<0.0001$ ) 
a

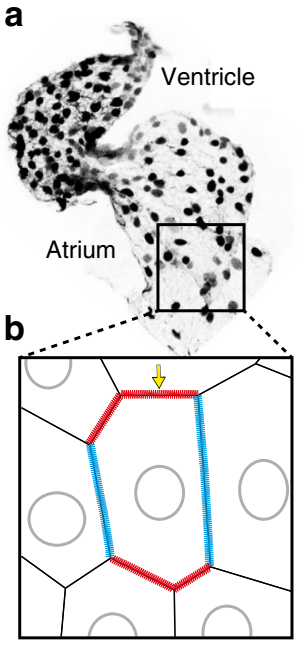

Pre-dissection

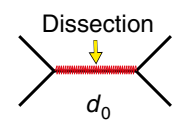

Post-dissection

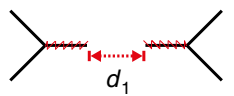

C

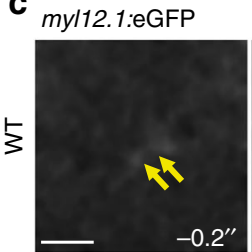

Pre-dissection

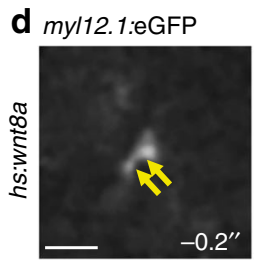

Pre-dissection
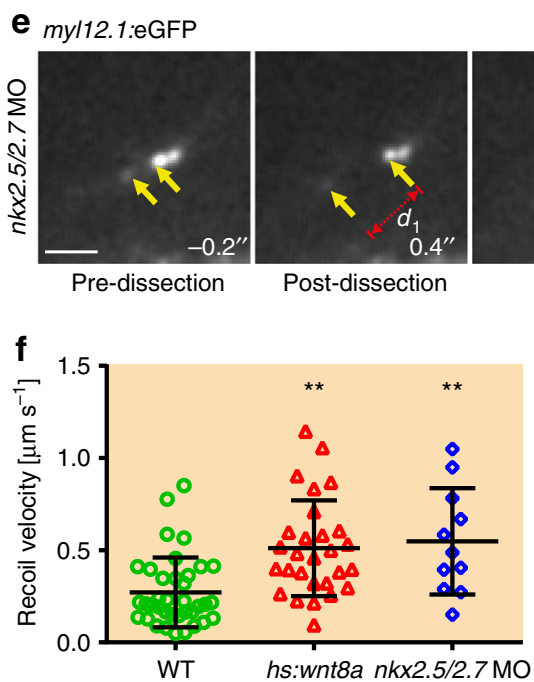

Post-dissection

Post-dissection

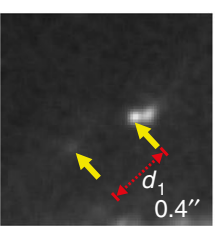

WT
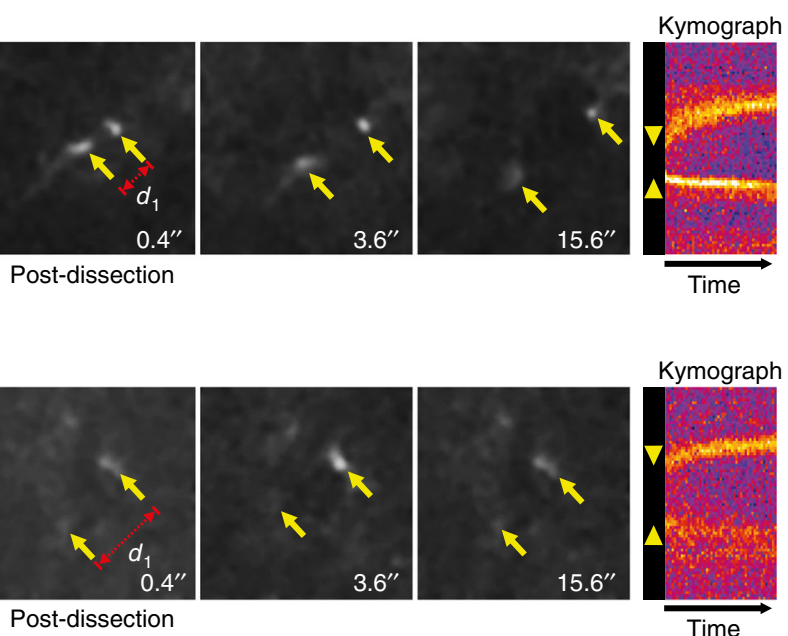

Time
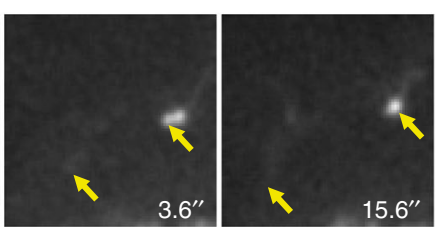

Kymograph

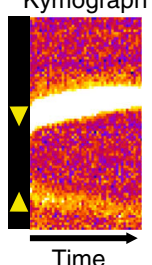

Fig. 4 Endocardial tissue tension increases upon Wnt8a overexpression or loss of Nkx2.5/Nkx2.7. a, b Schematic model illustrating the endocardial region in which the laser cuts were performed. Local laser cut (yellow arrow) of the subcortical actomyosin network within the shorter membrane compartment (red) which is oriented perpendicular to the direction of intra-cardiac blood flow at time point $d_{0}$ causes an actomyosin recoil ( $d_{1}$ ). c-e Time lapse sequences following a laser cut within the endocardium of $40 \mathrm{hpf}$ embryos with the $\mathrm{Tg}$ (act2:myl12.1-EGFP)e2212 transgenic reporter that marks the actomyosin network. Yellow arrows show the actomyosin recoil distance within 0.4 ms upon laser dissection. Kymographs indicate the temporal recoil of the actomyosin network along the membrane compartment where the laser cut was performed. The recoil of the open junction ends is visualized with the fluorescence intensity and the initial opening of the junction is marked by yellow arrowheads. $\mathbf{d}$, e Time lapse analyses demonstrate a larger opening of the junction $\left(d_{1}\right)$ and a faster recoil of the actomyosin network (yellow arrowheads in the kymograph) upon Wnt8a overexpression or loss of Nkx2.5/Nkx2.7 following laser cuts. Scale bars, $20 \mu \mathrm{m}$. f Comparison of initial recoil velocities ( $\mu \mathrm{m}$ per sec) which are lower in WT ( $n=37$ hearts) compared with Wnt8a overexpressing embryos ( $n=28$ hearts) or upon loss of Nkx2.5/Nkx2.7 ( $n=11$ hearts). Mean values \pm SD are shown. One-way ANOVA was used to compare each condition with WT $\left({ }^{\star \star} p<0.001\right)$

non-muscle myosin II. Time lapse movies of $T g$ (act2:myl12.1$E G F P)^{22212}$ localization after the cell junction cuts were recorded for a period of $15.6 \mathrm{~s}$ at 0.2 second intervals (Fig. 4c-e; Supplementary Movies 4-6). Upon Wnt8a overexpression, tensile forces in endocardial cell junctions are higher compared with WT (Fig. 4c), as indicated by a greater width of myosin recoil at $0.4 \mathrm{~s}$ after laser dissection (Fig. 4d). Based on slope measurements of the kymographs (see Materials and Methods), we determined the initial recoil velocities ( $\mu \mathrm{m}$ per sec) in WT (Fig. 4c), upon Wnt8a overexpression (Fig. 4d), or upon loss of Nkx2.5/Nkx2.7 (Fig. 4e). These analyses revealed that endocardial cell junctions are under higher tensile forces while chamber dimensions are expanding (Fig. 4f). Our findings imply that the expanding dimensions of atrial myocardial chambers cause stronger tensile forces upon cell junctions within the endocardium. This finding suggested that in response, endocardial cells may activate biomechanical signaling pathways to regulate proliferation, which subsequently releases tension within this tissue.

Cadherin-5 mediates endocardial proliferation during chamber expansion. The sensing and transmission of force between endothelial cells has been attributed to a mechanosensitive complex that includes Cadherin- $5^{20-23}$. This endothelial-specific adherens junction protein links the contractile acto-myosin cytoskeleton to the cell membrane ${ }^{21,24}$. We wondered whether Cadherin-5 plays a similar role within endocardium and examined whether a loss of Cadherin-5 would reduce endocardial cell numbers upon the overexpression of Wnt8a or loss of Nkx2.5. Since cadherin- 5 mutants display a dysmorphic heart ${ }^{24}$ and lack blood flow, which would impact endocardial proliferation ${ }^{4}$ we 

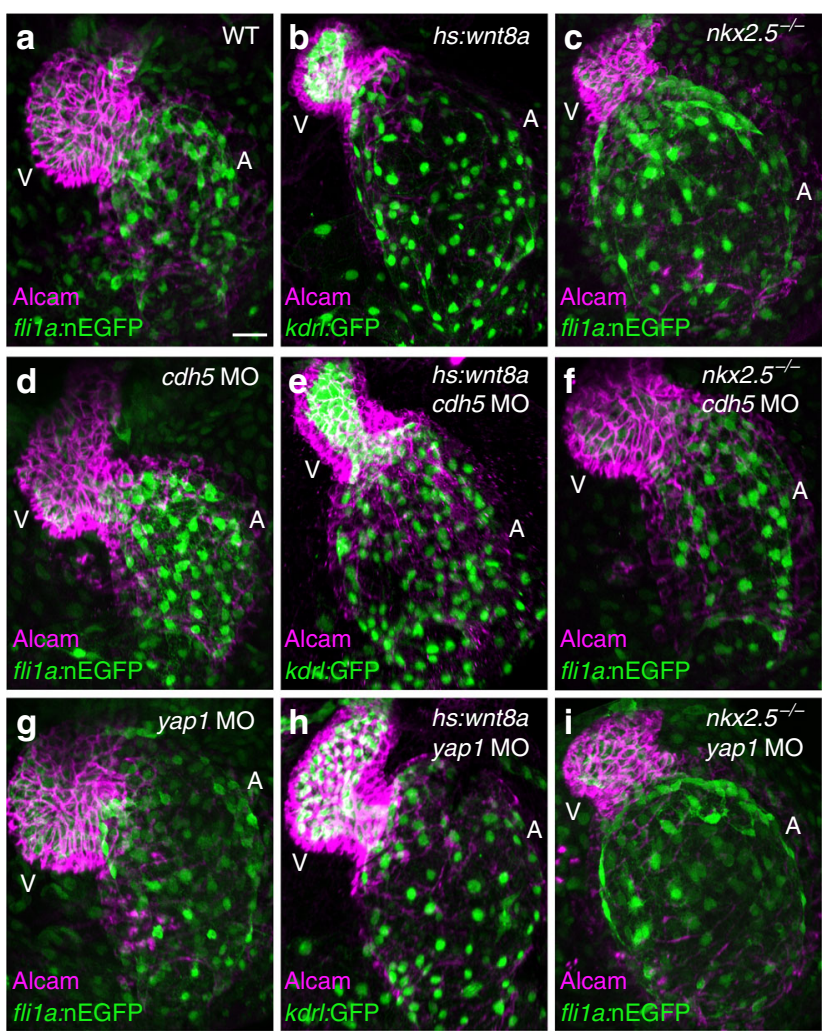
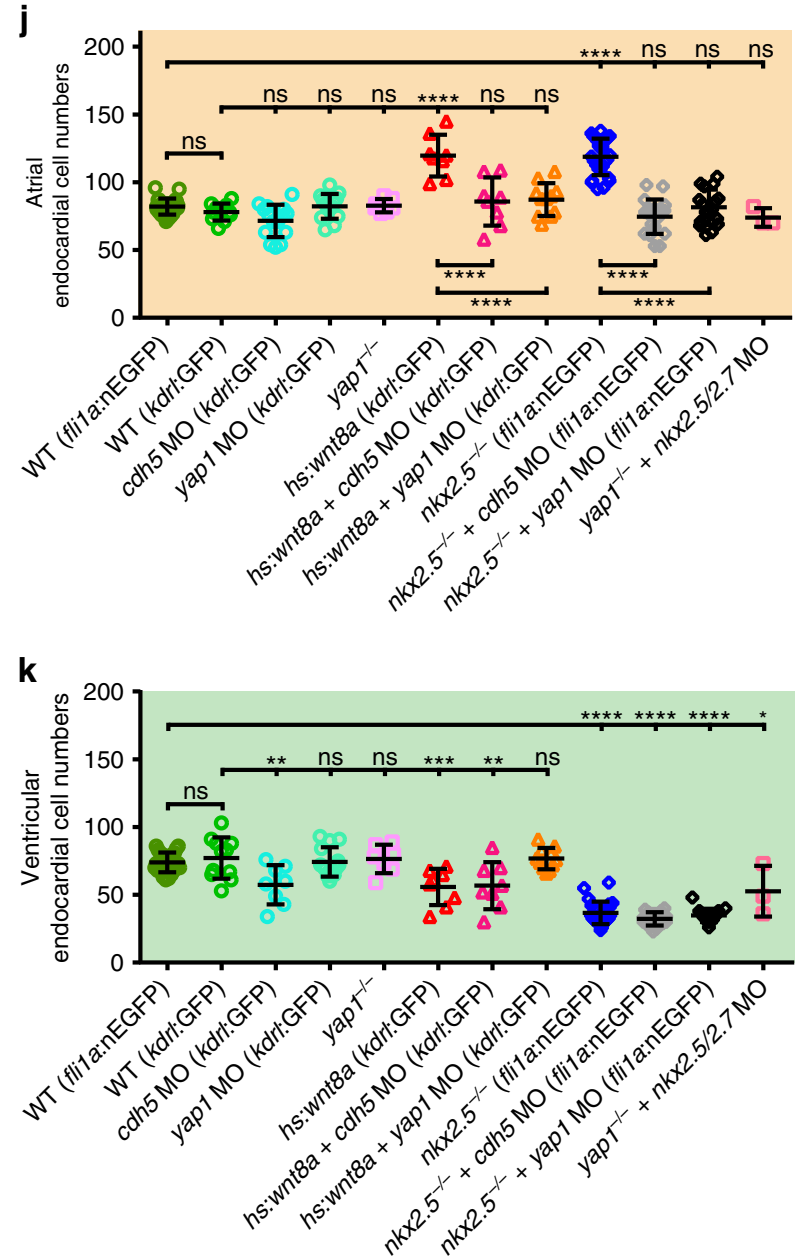

Fig. 5 Loss of Cadherin-5 or Yap1 prevents endocardial cell number increases during atrial chamber expansion. a-i Reconstructions of confocal z-stacks of

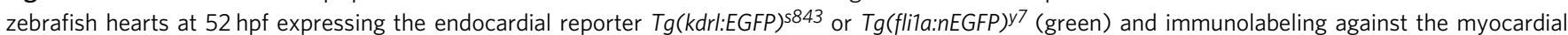
marker Alcam (magenta). j Quantifications of endocardial cell numbers at $52 \mathrm{hpf}$. b, j Upon Wnt8a overexpression ( $n=7$ hearts), atrial endocardial cell numbers increase. $\mathbf{c}, \mathbf{j}$ Similarly, in $n k \times 2.5^{\mathrm{vu} 179}$ mutants $(n=27$ hearts), endocardial cell numbers increase within the atrium. $\mathbf{d}, \mathbf{j}$ MO-mediated knock down of Cadherin-5 (Cdh5) ( $n=7$ hearts) does not cause a reduction of endocardial cell numbers within the atrium. $\mathbf{e}, \mathbf{j}$ Loss of Cdh5 prevents increased atrial endocardial cell numbers upon overexpression of Wnt8a ( $n=8$ hearts). $\mathbf{f}, \mathbf{j}$ Loss of Cdh5 suppresses increased atrial endocardial cell numbers in $n k \times 2.5^{\mathrm{vu} 179}$ mutants ( $n=19$ hearts). $\mathbf{g}$, j Loss of Yap1 via MO-mediated knock down ( $n=14$ hearts) or in yap $7^{f u 48}$ mutants (j, $\left.\mathbf{k}\right)$ does not affect ventricular or atrial endocardial cell numbers. $\mathbf{h}$, $\mathbf{j}$ Knock down of Yap1 in Wnt8a overexpressing embryos normalizes ventricular and atrial endocardial cell numbers ( $n=8$ hearts). $\mathbf{i}$, $\mathbf{j}$ Loss of Yap1 rescues atrial endocardial cell numbers in $n k \times 2.5^{\mathrm{vu}} 179$ mutants ( $n=17$ hearts). $\mathbf{j}$ A loss of Nkx2.5/Nkx2.7 in yap 7 fu48 mutants also rescues atrial endocardial cell numbers. A atrium, $\vee$ ventricle. Scale bars, $30 \mu \mathrm{m}$. j, $\mathbf{k}$ Quantifications of endocardial cell numbers in atrium and ventricle. Mean values \pm SD are shown. Two-way ANOVA was used to compare each condition with its WT control in each individual chamber (ns not significant; ${ }^{* \star} p<0.01 ;{ }^{* \star *} p<0.001 ;{ }^{\star \star \star \star *} p<0.0001$ )

used a well-established antisense oligonucleotide morpholino (MO) against Cadherin- $5^{25}$ and selected those embryos with blood flow for further analyses. In support of an effect of Cadherin-5 on cell proliferation during chamber expansion, we found that atrial endocardial cell numbers were significantly reduced upon MO-mediated knock down of Cadherin-5 in Wnt8a overexpressing or $n k x 2.5^{\text {vul79 }}$ mutant but not in WT hearts (Fig. 5a-f, j). Immunohistochemical analysis revealed that Cadherin-5 was localized to endocardial cell junctions in Wnt8a overexpressing or $n k x 2.5^{\text {vul79 }}$ mutant embryos in a manner similar to WT (Supplementary Fig. 5). Within the ventricular endocardium, loss of Cadherin-5 caused diminished cell numbers (Fig. 5k), which implies that Cadherin-5 also has a physiological role in WT ventricular endocardial cell proliferation. In tune with this finding, EdU-based proliferation assays revealed that a loss of Cadherin-5 strongly diminished endocardial cell proliferation under these different conditions (Supplementary Fig. 6). To test whether this effect was due to a loss of cell junctional tensile forces upon loss of Cadherin-5, we next performed UV-laserbased force measurements (as described above) which showed that a loss of Cadherin-5 has no effect (Supplementary Fig. 7; Supplementary Movie 7). Taken together, these results suggest a role for Cadherin-5 in force sensing/transmission but not in the generation of cell junctional tensile forces within the endocardial layer during the atrial chamber expansion process.

Yap1 is required during atrial endocardial chamber expansion. Hippo signaling is involved in the control of tissue/organ size and cell proliferation in a manner that is functionally dependent on mechanical tension ${ }^{26}$. The Hippo pathway transcription factor Yap1 plays a role in the control of endothelial cell proliferation in response to blood flow 27,28 . We used two independent means of detecting Yap1 localization within the endocardium: First, 

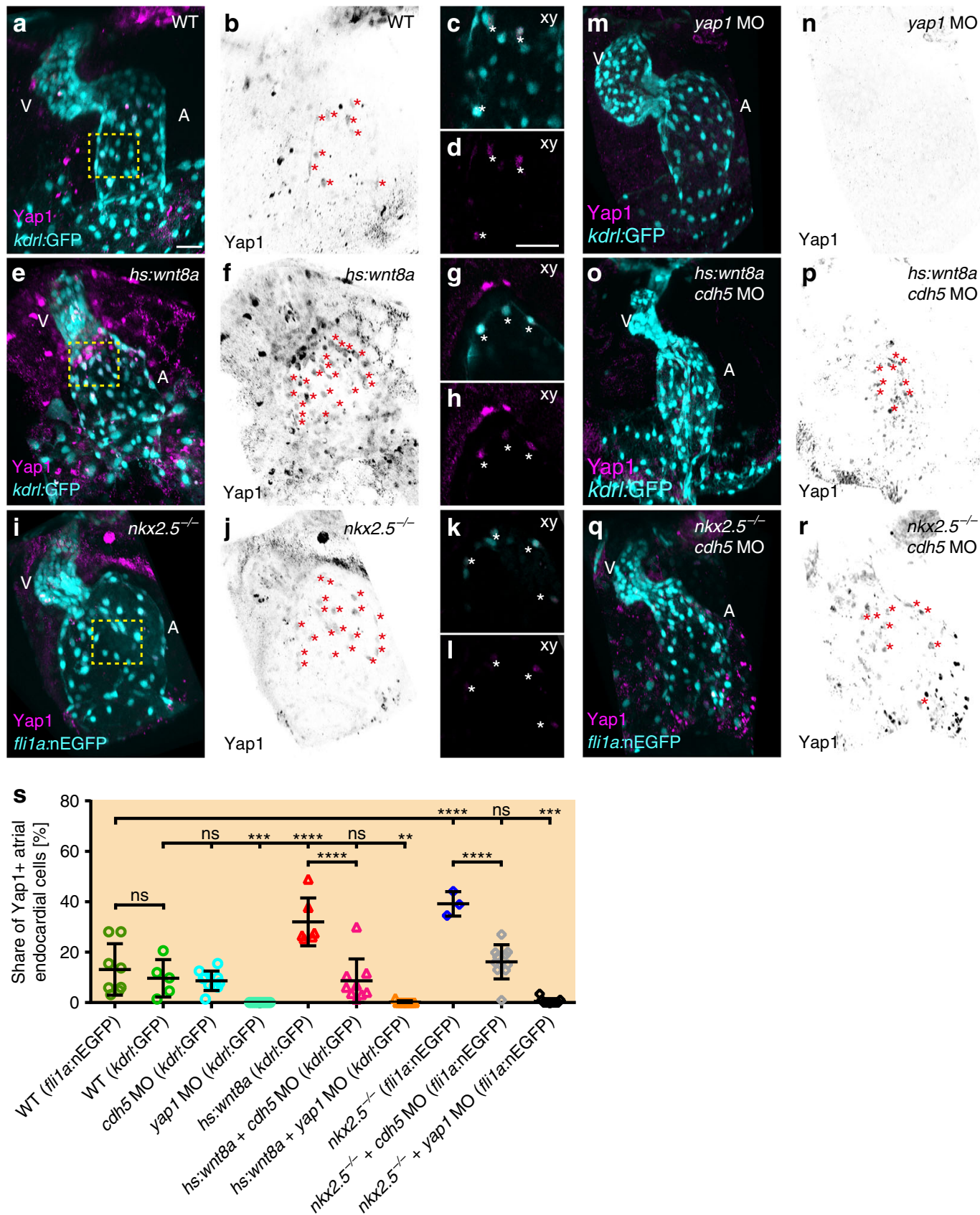

Fig. 6 Yap1 nuclear localization within the atrial endocardium increases upon chamber expansion. $\mathbf{a}, \mathbf{e}, \mathbf{i}, \mathbf{m}, \mathbf{0}$, q Reconstructions of confocal z-stacks of zebrafish hearts at $52 \mathrm{hpf}$ expressing the endocardial reporters $\mathrm{Tg}(\mathrm{kdrl}: E G F P){ }^{s 843}$ or $\mathrm{Tg}$ (fli7a:nEGFP)y7 (cyan) and immunolabeling against zebrafish Yap1 (magenta). b, f, j, $\mathbf{n}, \mathbf{p}, \mathbf{r}$ Yap1 immunolabeling is inverted in black/white and endocardial cells which show co-localization with Yap1 labeling are marked with red asterisks. A atrium, $V$ ventricle. Scale bars, $30 \mu \mathrm{m} . \mathbf{c}, \mathbf{d}, \mathbf{g}, \mathbf{h}, \mathbf{k}, \mathbf{I}$ Magnifications of single confocal $X Y$ section planes (yellow box in $\mathbf{a}, \mathbf{e}, \mathbf{i}$ ) are shown in $\mathbf{c}, \mathbf{g}$, $\mathbf{k}$ and, in $\mathbf{d}, \mathbf{h}, \mathbf{l}$, only Yap1 immunolabeling is shown. Endocardial cells, labeled by $T g(k d r l: E G F P)^{5843}$ or Tg(flita:nEGFP)y7, that show colocalization with Yap1 are indicated with an asterisk. Scale bars, $30 \mu \mathrm{m}$. s Quantifications of the share of Yap1-positive endocardial cells relative to the total number of endocardial cells within the atrium. Upon Wnt8a overexpression ( $n=6$ hearts) or loss of Nkx2.5 ( $n=3$ hearts), the share of Yap1-positive endocardial cell numbers significantly increases within the developing atrial endocardium. Loss of Cadherin-5 (Cdh5) changes the share of Yap1-positive endocardial cells among Wnt8a overexpressing ( $n=9$ hearts) or $n k x 2.5^{\text {vul79 }}$ mutant embryos ( $n=10$ hearts) to WT levels. Knock down of Yap1 in all conditions leads to a massive reduction of Yap1-positive endocardial cells within the atrium (yap1 MO: $n=11$ hearts; yap1 MO + hs:Wnt8a: $n=9$ hearts; yap1 $\mathrm{MO}+n k \times 2.5^{\mathrm{vu} 179}$ mutant: $n=11$ hearts). Mean values \pm SD are shown. One-way ANOVA was used to compare each condition with its WT control (ns not significant, ${ }^{\star \star} p<0.01 ;{ }^{* \star \star} p<0.001 ;{ }^{\star \star \star \star} p<0.0001$ )

immunolabeling with a Yap1-specific antibody revealed Yap1positive endocardial cells within both heart chambers at $52 \mathrm{hpf}$ (Fig. 6a-d; Supplementary Fig. 8a-d) which was strongly reduced in yap1 morphants (Fig. $6 \mathrm{~m}, \mathrm{n}$ ). Second, a transgenic Yap-specific reporter line, $\operatorname{Tg}(\text { fli1a:EGFP-YAP })^{n c v 35}$, which also indicated subcellular localization of Yap1 within endocardial cells (Supplementary Fig. 8e-h). To elucidate whether Yap1 functionally contributes to higher atrial endocardial cell numbers under conditions of increased tissue tension, we performed a MOmediated knock down when Wnt8a was overexpressed or in 
$n k \times 2.5^{\text {vu179 }}$ mutants (Fig. 5a-c, g-i, j, k). Although knock down of Yap1 and yap1 $1^{\text {fu } 48}$ mutant endocardium and myocardium were morphologically indistinguishable from WT and had blood flow (Fig. 5g, j, k; Supplementary Fig. 9), a knock down of Yap1 under conditions of Wnt8a overexpression or loss of Nkx2.5, as well as the effect of the yap1 $1^{\text {fu}} 48$ mutation upon loss of $\mathrm{Nkx} 2.5$ / Nkx2.7 significantly reduced endocardial cell numbers (Fig. 5j, k) and reduced atrial endocardial cell proliferation as indicated by an EdU-incorporation assay (Supplementary Fig. 6). This demonstrates an important role of Yap1 in endocardial cell proliferation control when atrial chamber dimensions strongly expand and generate tension on the tissue.

Cadherin-5 triggers Yap1 nuclear translocation within endocardium. Increased tissue tension causes Yap1 translocation into the nucleus, where it promotes the expression of genes involved in cell proliferation ${ }^{28}$. E-Cadherin, for instance, was shown to induce cell cycle reentry and sequential activation of Yap1 dependent on mechanical strain ${ }^{29}$. We used the Yap1-specific antibody and assessed the ratio of endocardial cells with Yap1 nuclear localization relative to the entire population of atrial endocardial cells (Fig. 6). Next, we analyzed the subcellular Yap1 localization under the two conditions with increased tissue tension. We observed a significant increase in the share of atrial endocardial cells with nuclear Yap1 localization when overexpressing Wnt8a (Fig. 6e-h) or having lost Nkx2.5 (Fig. 6i-l) compared to WT (Fig. 6a-d, s). This suggests that the increase in proliferation in both genetic conditions is due to the localization of Yap1 to the nucleus.

Given the important role of Cadherin- 5 in force transmission within the endocardial tissue layer, we also tested whether it was required for Yap1 nuclear translocation upon overexpression of Wnt8a or in $n k \times 2.5^{\text {vul79 }}$ mutants. Consistent with such a model, loss of Cadherin-5 reduced the share of atrial endocardial cells with a nuclear localization of Yap1 in these two conditions (Fig. $60-s)$. These findings imply that Cadherin-5-mediated force sensing/transmission is required for the nuclear localization of Yap1. Another implication is that Yap1-dependent proliferation is one mode by which tensile forces within endocardial tissue are reduced during chamber expansion.

\section{Discussion}

Here, we used two different genetic conditions to model atrial myocardial chamber expansion and to study the capability of the endocardium to adapt to this enhanced myocardial growth. In summary, our work suggests that some mode of intra-organ communication between myocardium and endocardium serves to coordinate the growth of different tissues of the heart during cardiac ballooning. Our data suggests that the expansion of the myocardial chambers is transmitted to the endocardium. As a result, junctional forces rise within the endocardial tissue layer. Here, we propose a model whereby these forces are transduced via Cadherin-5 through the nuclear localization of Yap1, a transcription factor that stimulates proliferative signaling (Fig. 7). The means by which myocardial chamber expansion is communicated between the two tissue layers is currently unknown but may involve biomechanically active extracellular matrix components, which are deposited within the tight space between endocardium and myocardium ${ }^{2,3}$. Other means of intra-organ communication are also possible which would result in increased tissue tension within the endocardium. An alternative explanation might be that the mechanical strain due to myocardial chamber expansion is transmitted by cell junctional contacts between myocardial and endocardial cells. Just as the adaptive growth of the endocardium increases through an increase of these forces, it may end as a

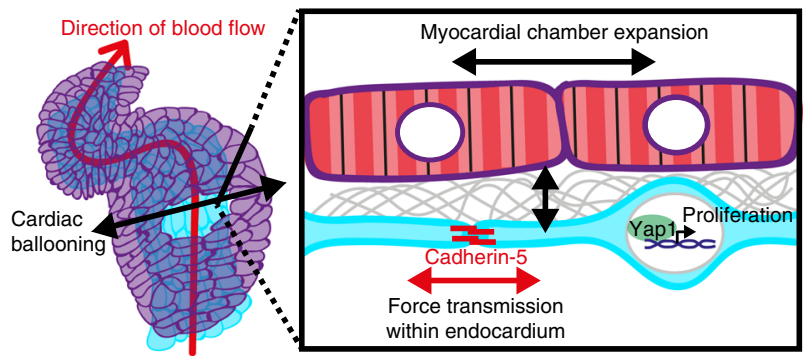

Fig. 7 Model of intra-organ communication within the developing zebrafish heart. Myocardial chamber expansion causes increased tensile forces within the endocardial cell junctions which are transmitted by Cadherin- 5 . This in tune causes the nuclear translocation of the Hippo signaling transcription factor Yap1 activating cell proliferation within endocardial cells

result of the relaxation of tensile forces on the tissue. Conversely, we also find that smaller myocardial ventricular chamber dimensions correspond with lower endocardial cell numbers. This finding suggests that this mechanism not only impacts expanding chambers but also holds true under physiological conditions of the ventricle.

The developing heart experiences many other modes of intraorgan communication based on chemical signaling ${ }^{2,3}$. How these diverse modes of signaling are integrated at the cellular and tissue levels remains to be addressed. The activation of proliferation within the developing endocardium in response to changes in chamber dimensions has important implications not only for our understanding of heart morphogenesis during development but also for characterizing patho-physiological conditions of the heart. It will be of great interest to revisit endocardial chamber development under conditions of congenital heart defects. The present work provides a further component in this repertoire of intra-organ communication, which causes biomechanical signaling and tensile force-triggered proliferation within the endocardium. This pattern may also hint at a general blueprint for the coordination of growth rates in the tissues of other complex organs; it shows that homeostatic responses which arise through the morphological adaptations in size of one tissue likely coordinate tissue-intrinsic growth rates within other tissues as well.

\section{Methods}

Zebrafish handling and lines. Handling of zebrafish was done in compliance with German, Brandenburg and Lower Saxony state law, carefully monitored by the local authority for animal protection (LAGV, Brandenburg and LANUV, Lower Saxony). The following strains of zebrafish were maintained under standard conditions as previously described ${ }^{30}: T g(k d r l: E G F P)^{s 84331}, T g(m y l 7: E G F P)^{t w u} 3432$, Tg(fli1a:nEGFP)y 33 $\mathrm{Tg}(\text { flt1:YFP })^{\text {hu4624 15 }}, \mathrm{Tg}$ (hsp70l:Wnt8a-GFP)w34 34, Tg(act2: myl12.1-EGFP) $)^{e 2212}$ 19, Tg(fli1:EGFP-YAP $)^{n c v 3527}, \mathrm{nkx} 2.5^{\text {vu179 } 12}$, and yap $1^{\text {fu4 } 4835}$.

Antisense oligonucleotide Morpholinos. Knock down studies were performed by injection of the following antisense oligonucleotide morpholinos (MO) (Gene Tools, LLC):

MO1-cadherin-525: 5'-TTTACAAGACCGTCTACCTTTCCAA-3

yap1-ATG $\mathrm{MO}^{36}: 5^{\prime}$-AGCAACATTAACAACTCACTTTAGG-3'

nkx2.5 ATG $\mathrm{MO}^{13}: 5^{\prime}$-TCATTTGGCTAGAGAACATTGCCAT-3

nkx2.7 ATG MO ${ }^{13}: 5^{\prime}$-TGGAGGTCACAGGACTCGGAAGCAT-3'

A total of $1 \mathrm{nl}$ MO solution was injected into one-cell-stage embryos at the following concentration: $1 \mathrm{ng}$ yap1 $\mathrm{MO}, 0.5 \mathrm{ng} c d h 5 \mathrm{MO}, 4 \mathrm{ng} n k x 2.5 \mathrm{MO}$, and $2 \mathrm{ng}$ $n k x 2.7$ MO.

Transgenic overexpression via heat shock. For heat shock induced overexpression of $\mathrm{Tg}\left(\mathrm{hspl}\right.$ l0:Wnt8a-GFP) ${ }^{w 34}$ up to 50 embryos were collected in a $2 \mathrm{ml}$ Eppendorf tube and pre-warmed egg water $\left(38^{\circ} \mathrm{C}\right)$ was added at $24 \mathrm{hpf}$. Embryos were incubated at $38^{\circ} \mathrm{C}$ for $45 \mathrm{~min}$ in a Thermomixer Comfort, which was turned to the side while slowly shaking at $350 \mathrm{rpm}$. After heat shock, embryos were transferred to Petri dishes filled with egg water and further incubated at $28.5^{\circ} \mathrm{C}$. At $30 \mathrm{hpf}$, GFP-positive embryos, carrying the transgenes, were selected and separated from GFP-negative fish (used as controls) and further incubated until $52 \mathrm{hpf}$. 
Immunohistochemistry. Embryos at the desired developmental stage were first anesthetized with $0.2 \%$ tricaine for one minute followed by fixation with $4 \%$ paraformaldehyde (PFA) for $1 \mathrm{~h}$ at room temperature. Fixed samples were washed for 30 min with PBT which was followed by blocking with $10 \%$ NGS (Normal Goat Serum) plus $2 \mathrm{mg}$ per $\mathrm{ml}$ BSA and $0.8 \%$ Triton-X100 for $2 \mathrm{~h}$ at room temperature. For immunolabeling of the cell surface marker Alcam or Yap1 blocking was performed with PBDT (PBS $1 \times+0.1 \%$ v/v Tween $20+1 \%$ DMSO) supplemented with $10 \%$ NGS. The primary antibody was incubated at $4{ }^{\circ} \mathrm{C}$ overnight in PBT containing $0.8 \%$ Triton-X100. The following primary antibodies were used in this study: anti-Islet1/2 (1:10; 39.4D5, Developmental Studies Hybridoma Bank), antiIslet1 (1:2000, Genetex, USA), anti-Alcam (1:100; zn-8, Developmental Studies Hybridoma Bank), anti-Yap1 (1:200, generated in the Lecaudey lab), anti-GFP (1:500, Aves labs, USA, \#GFP-1020) and anti-atrial myosin heavy chain (1:10; S46, Developmental Studies Hybridoma Bank). After incubation samples were washed every $30 \mathrm{~min}$ for at least $2 \mathrm{~h}$. This step was followed by secondary antibody incubation overnight at $4{ }^{\circ} \mathrm{C}$. The following secondary antibodies were used: Alexa Fluor 647 goat anti-mouse IgG (1:250; Life Technologies), Alexa Fluor 647 goat anti-rabbit IgG (1:250; Life Technologies), and goat anti-chicken FITC (1:250, Aves labs, \#F-1005). In addition, the following dyes were incubated in combination with the secondary antibody: Alexa Fluor 546 Phalloidin (1:100, Invitrogen) and DAPI (1:1000, Sigma). GFP protein within the transgenic reporter $\mathrm{Tg}$ (fli1:EGFP-YAP) $n c v 3527$ was visualized via an anti-GFP immunolabeling. Specimens were mounted in PBT and imaged on Leica TCS SP8 confocal laser microscope.

EdU labeling and quantification of cell proliferation. Myocardial or endocardial proliferation in embryos expressing $\mathrm{Tg}(m y l 7: E G F P)^{t w u 34}, \mathrm{Tg}(\mathrm{kdrl}: E G F P)^{s 843}$, or $\mathrm{Tg}$ (flila:nEGFP) $)^{7}$ was assessed using the Click-iT EdU Imaging Kit (Life Technologies) following the previous reported protocols ${ }^{37}$. Anaesthetized, dechorionated embryos at $30 \mathrm{hpf}$ were injected with $2 \mathrm{nl}$ EdU solution (100 $\mu \mathrm{M}$ EdU, 2\% DMSO, $0.1 \%$ phenol red) into the circulatory system. Incubation was performed until 52 hpf, afterwards embryos were rinsed with egg water and fixed in 4\% PFA over night at $4^{\circ} \mathrm{C}$. In order to label the $\mathrm{Tg}(m y l 7: E G F P)^{t w u 34}$ and $\mathrm{Tg}(\mathrm{kdrl}: E G F P)^{s 843}$ or $\mathrm{Tg}(\mathrm{fli1a}$ : $n E G F P)^{y 7}$ reporter lines a whole-mount immunostaining was performed using chicken anti-GFP (1:500, Aves labs, USA, \#GFP-1020) as the primary antibody and goat anti-chicken FITC (1:250, Aves labs, \#F-1005) as a secondary antibody. The Click-iT reaction for EdU staining was performed by following the manufacturer's instructions. Images were acquired with a Leica TCS SP8 confocal laser microscope using a $20 \times$ water-immersion objective. Quantification of either proliferative myocardial or endocardial cells was performed using Imaris (Bitplane, UK). Cells were counted as proliferative when double labeled with GFP and EdU. The share of EdU-positive cells was calculated as the percentage of proliferative cells among the total number of myocardial or endocardial cells, respectively.

Multi-color mosaic-labeling. To label the myocardium in a mosaic color fashion in vivo, myl7:TagRFP-T and myl7:TagBFP constructs were injected (kindly provided by $D$. Stainier $)^{16}$ together with transposase mRNA into one cell stage transgenic $T g(m y l 7: E G F P)^{t w u 34}$ or double transgenic $T g(h s p 70 l: W n t 8 a-G F P)^{w 34}$; $T g$ $(m y l 7: E G F P)^{t w u 34}$ embryos. After Tol2-mediated integration a clonal expression pattern of the constructs was created and the myocardium was labeled with a mixture of three fluorescent proteins. For live high-resolution imaging individual hearts of each condition with a colorful expression pattern were selected and mounted in $1 \%$ low-melting agarose supplemented with $0.4 \mathrm{mg}$ per ml Tricaine (3amino benzoic acidethylester; Sigma-Aldrich, A-5040) to stop the heartbeat while the images were taken. An image stack was acquired on a confocal laser scanning microscope Leica TCS SP8 with a $\times 20$ objective every 30 min from $48-52$ hpf with optical sections at $1 \mu \mathrm{m}$ thickness. Time lapse movies and images were analyzed using Imaris Image Analysis Software (Bitplane, UK).

Microscopy and image analysis. Images were acquired using either a Leica SP8 confocal laser microscope or a Leica SP5 confocal laser microscope with $\times 20$ or $\times 25$ magnification for whole mount hearts, respectively. For fixed samples, optical sections with $1 \mu \mathrm{m} \mathrm{z}$-steps were generated. For live-imaging, images were taken every $30 \mathrm{~min}$ for up to $5 \mathrm{~h}$ with optical sections of $1 \mu \mathrm{m}$ z-step thickness. Maximal intensity projections were generated using the same settings for all samples. All images were processed and analyzed using Fiji (NIH, USA) or Imaris (Bitplane, UK). Brightness and contrast were adjusted with Imaris and Fiji.

Quantifications of Yap1-positive endocardial cell numbers. To quantify endogenous Yap1, endothelial/endocardial $T g(k d r l: E G F P)^{s 843}$ or $T g(f l i 1 a: n E G F P)^{y 7}$ reporter lines were immunolabeled and DAPI was used as a nuclear marker. Cells doubly positive for nuclear Yap1 immunolabeling and an endothelial/endocardial transgene were counted as a Yap1-positive endocardial cell. The share of Yap1positive cells was calculated as the percentage of Yap1-positive cells among the total number of endocardial cells. Images were acquired on a Leica SP8 confocal laser microscope and analyzed using Imaris (Bitplane, UK).

Quantification of cell numbers and cell size measurements. Images were acquired on a Leica SP8 confocal laser microscope and processed with Imaris
(Bitplane, UK). Maximal intensity projections were made to count the number of myocardial or endocardial cells, respectively. To count myocardial cells, the transgenic reporter $\operatorname{Tg}(m y l 7: G F P)^{t w u} 34$ or/and an immunohistochemistry staining against Alcam was acquired. Since the reporter line labels myocardial nuclei, antiAlcam staining was performed to mark cell outlines. To count endocardial cells, the transgenic reporter line $\operatorname{Tg}(k d r l: G F P)^{s 843}$ or $\operatorname{Tg}(f l i 1 a: n E G F P)^{y 7}$ was used. To specifically count cells within individual heart chambers, a counter staining of the myocardial atrium was performed using anti-Myosin heavy chain 6 (Myh6) immunolabeling.

Imaris was used to measure distances between two neighboring nuclei and at least 20 different length measurements were performed per chamber. Myocardial cell surface areas were quantified using Fiji (NIH, USA). Myocardial cell borders were immunolabeled with anti-Alcam. Cell surroundings were measured in Fiji using the free draw tool. Cell surface areas are displayed in $\mu \mathrm{m}^{2}$. For each heart at least 15 cells were analyzed.

Zebrafish endocardial tissue tension measurements. Endocardial tissue tension was measured at $40 \mathrm{hpf}$ using a UV-laser dissection set up as previously described $^{20}$. First, embryos were anesthetized with $0.2 \%$ Tricaine and mounted in $1 \%$ agarose containing Tricaine to pause the heartbeat. Using a Zeiss $63 \times 1.2 \mathrm{NA}$ water immersion lens, $4 \mu \mathrm{m}$ long cuts were generated with 25 pulses of $1000 \mathrm{~Hz}$. Fluorescent images were acquired with a iXon DU-897-BV camera (Andor Technology) using exposure times of $200 \mathrm{~ms}$ and a loop time of $200 \mathrm{~ms}$. To ensure comparability of the measurements, each heart was cut only once to avoid effects on the entire heart due to the release of tissue tension and only cuts without any immediate wound response were analyzed. For analysis, cells located within the lower part of the endocardial inflow tract were targeted. Within each cell, a short membrane compartment was cut which was oriented perpendicular to intracardiac blood flow or a long membrane compartment was cut oriented parallel to blood flow. Due to the findings that both membrane compartments show comparable initial recoil velocities, only the perpendicular membrane compartments were selected for reliable comparability. Quantification of the initial recoil velocity upon junction dissection was performed as previously described using Fiji $(\mathrm{NIH}$, USA) ${ }^{38,39}$ : A kymograph was generated to specifically display the myl12.1:eGFP signal along the junction versus time and the opening slope was determined after dissection. The tissue tension at endocardial cell junctions was calculated by linearregression analysis of the initial relaxation velocity. For statistical analysis PRISM Graph Pad software was used.

Statistical analyses. If not stated otherwise, all experiments in this study were performed in at least three independent biological replicates. Statistical analysis was carried out using PRISM Graph Pad software. For comparison of two groups, that were parametrically distributed, an unpaired two-tailed Student's $t$-test was performed. In order to compare more than two groups, a one-way ANOVA test and, for two categorical independent variables, a two-way ANOVA was performed. A $p$-value of $\leq 0.05$ was considered significant in all experiments $\left({ }^{*} p \leq 0.05 ;{ }^{* *} p \leq 0.01\right.$; $\left.{ }^{* * *} p \leq 0.001 ;{ }^{* * * *} p \leq 0.0001\right)$.

Reporting summary. Further information on research design is available in the Nature Research Reporting Summary linked to this article.

\section{Data availability}

The authors declare that the data supporting the findings of this study are available within the paper and its supplementary information files. The rest of the data are available from the authors upon reasonable request.

Received: 8 January 2019 Accepted: 8 August 2019 Published online: 11 September 2019

\section{References}

1. Luxán, G., D’Amato, G. \& de la Pompa, J. L. in Etiology and Morphogenesis of Congenital Heart Disease(eds Nakanishi T., Markwald R., Baldwin H., Keller B., Srivastava D., Yamagishi H.) Ch. 12 (Springer, Tokyo, 2016).

2. Staudt, D. \& Stainier, D. Uncovering the molecular and cellular mechanisms of heart development using the Zebrafish. Annu. Rev. Genet. 46, 397-418 (2012).

3. Haack, T. \& Abdelilah-Seyfried, S. The force within: endocardial development, mechanotransduction and signalling during cardiac morphogenesis. Development 143, 373-386 (2016).

4. Dietrich, A.-C., Lombardo, V. A., Veerkamp, J., Priller, F. \& AbdelilahSeyfried, S. Blood flow and Bmp signaling control endocardial chamber morphogenesis. Dev. Cell 30, 367-377 (2014).

5. de Pater, E. et al. Distinct phases of cardiomyocyte differentiation regulate growth of the zebrafish heart. Development 136, 1633-1641 (2009). 
6. Zhou, Y. et al. Latent TGF- $\beta$ binding protein 3 identifies a second heart field in zebrafish. Nature 474, 645-648 (2011).

7. Lazic, S. \& Scott, I. C. Mef2cb regulates late myocardial cell addition from a second heart field-like population of progenitors in zebrafish. Dev. Biol. 354, 123-133 (2011).

8. Hami, D., Grimes, A. C., Tsai, H.-J. \& Kirby, M. L. Zebrafish cardiac development requires a conserved secondary heart field. Development 138, 2389-2398 (2011).

9. Samsa, L. A. et al. Cardiac contraction activates endocardial Notch signaling to modulate chamber maturation in zebrafish. Development 142, 4080-4091 (2015).

10. Auman, H. J. et al. Functional modulation of cardiac form through regionally confined cell shape changes. PLoS Biol. 5, e53 (2007).

11. Dohn, T. E. \& Waxman, J. S. Distinct phases of Wnt $/ \beta$-catenin signaling direct cardiomyocyte formation in zebrafish. Dev. Biol. 361, 364-376 (2012).

12. Targoff, K. L. et al. Nkx genes are essential for maintenance of ventricular identity. Development 140, 4203-4213 (2013).

13. Targoff, K. L., Schell, T. \& Yelon, D. Nkx genes regulate heart tube extension and exert differential effects on ventricular and atrial cell number. Dev. Biol. 322, 314-321 (2008).

14. Colombo, S. et al. Nkx genes establish second heart field cardiomyocyte progenitors at the arterial pole and pattern the venous pole through Isl 1 repression. Development 145, dev161497 (2018).

15. Hogan, B. M. et al. ccbel is required for embryonic lymphangiogenesis and venous sprouting. Nat. Genet. 41, 396-398 (2009).

16. Staudt, D. W. et al. High-resolution imaging of cardiomyocyte behavior reveals two distinct steps in ventricular trabeculation. Development 141, 585-593 (2014).

17. Schwartz, M. A. Integrins and extracellular matrix in mechanotransduction. Cold Spring Harb. Perspect. Biol. 2, a005066-a005066 (2010).

18. Petridou, N. I., Spiró, Z. \& Heisenberg, C.-P. Multiscale force sensing in development. Nat. Cell Biol. 19, 581-588 (2017).

19. Behrndt, M. et al. Forces driving epithelial spreading in zebrafish gastrulation. Science 338, 257-260 (2012)

20. Maitre, J.-L. et al. Adhesion functions in cell sorting by mechanically coupling the cortices of adhering cells. Science 338, 253-256 (2012).

21. Schwartz, M. \& Conway, D. Lessons from the endothelial junctional mechanosensory complex. F1000 Biol. Rep. 4, 1-6 (2012).

22. Conway, D. E. et al. Fluid shear stress on endothelial cells modulates mechanical tension across VE-Cadherin and PECAM-1. Curr. Biol. 23, 1024-1030 (2013).

23. Coon, B. G. et al. Intramembrane binding of VE-cadherin to VEGFR2 and VEGFR3 assembles the endothelial mechanosensory complex. J. Cell Biol. 208, 975-986 (2015).

24. Sauteur, L. et al. Cdh5/VE-cadherin promotes endothelial cell interface elongation via cortical actin polymerization during angiogenic sprouting. Cell Rep. 9, 504-513 (2014).

25. Mitchell, I. C., Brown, T. S., Terada, L. S., Amatruda, J. F. \& Nwariaku, F. E. Effect of vascular cadherin knockdown on zebrafish vasculature during development. PLoS ONE 5, e8807 (2010).

26. Meng, Z., Moroishi, T. \& Guan, K.-L. Mechanisms of Hippo pathway regulation. Genes Dev. 30, 1-17 (2016)

27. Nakajima, H. et al. Flow-dependent endothelial YAP regulation contributes to vessel maintenance. Dev. Cell 40, 523-536 (2017). e6.

28. Wang, K.-C. et al. Flow-dependent YAP/TAZ activities regulate endothelial phenotypes and atherosclerosis. Proc. Natl Acad. Sci. 113, 11525-11530 (2016).

29. Benham-Pyle, B. W., Pruitt, B. L. \& Nelson, W. J. Mechanical strain induces Ecadherin-dependent Yap1 and -catenin activation to drive cell cycle entry. Science 348, 1024-1027 (2015).

30. Westerfield, M., Doerry, E., Kirkpatrick, A. E., Driever, W. \& Douglas, S. A. An on-line database for zebrafish development and genetics research. Semin. Cell Dev. Biol. 8, 477-488 (1997).

31. Jin, S.-W. Cellular and molecular analyses of vascular tube and lumen formation in zebrafish. Development 132, 5199-5209 (2005).

32. Huang, C.-J., Tu, C.-T., Hsiao, C.-D., Hsieh, F.-J. \& Tsai, H.-J. Germ-line transmission of a myocardium-specific GFP transgene reveals critical regulatory elements in the cardiac myosin light chain 2 promoter of zebrafish. Dev. Dyn. 228, 30-40 (2003).

33. Roman, B. L. et al. Disruption of acvrll increases endothelial cell number in zebrafish cranial vessels. Development 129, 3009-3019 (2002).
34. Weidinger, G., Thorpe, C. J., Wuennenberg-Stapleton, K., Ngai, J. \& Moon, R $\mathrm{T}$. The Sp1-related transcription factors sp5 and sp5-like act downstream of Wnt/ $\beta$-catenin signaling in mesoderm and neuroectoderm patterning. Curr. Biol. 15, 489-500 (2005).

35. Agarwala, S. et al. Amotl2a interacts with the Hippo effector Yap1 and the Wnt/ $\beta$-catenin effector Lef1 to control tissue size in zebrafish. eLife 4, e08201 (2015).

36. Skouloudaki, K. et al. Scribble participates in Hippo signaling and is required for normal zebrafish pronephros development. Proc. Natl Acad. Sci. 106 8579-8584 (2009).

37. Hesselson, D., Anderson, R. M., Beinat, M. \& Stainier, D. Y. R. Distinct populations of quiescent and proliferative pancreatic-cells identified by HOTcre mediated labeling. Proc. Natl Acad. Sci. 106, 14896-14901 (2009).

38. Rauzi, M., Verant, P., Lecuit, T. \& Lenne, P.-F. Nature and anisotropy of cortical forces orienting Drosophila tissue morphogenesis. Nat. Cell Biol. 10, 1401-1410 (2008)

39. Mayer, M., Depken, M., Bois, J. S., Jülicher, F. \& Grill, S. W. Anisotropies in cortical tension reveal the physical basis of polarizing cortical flows. Nature 467, 617-621 (2010).

\section{Acknowledgements}

We would like to thank Nina Armbrecht and Anne Kühnel for excellent technical assistance. We are grateful to Daniele Teduccio and Vanessa Homm for excellent fish husbandry. We are indebted to M. Affolter, D. Stainier, and G. Weidinger for sharing reagents. We thank all members of the Seyfried group for constructive feedback on the project and critical reading the paper. D.B. was supported by the Excellence cluster REBIRTH. S.A.-S. was supported by the Excellence cluster REBIRTH, SFB958, by Deutsche Forschungsgemeinschaft (DFG) projects SE2016/7-2 and SE2016/10-1 and by the DZHK. D.Y. was supported by NIH R01 HL108599 and NIH R01 HL069594.

\section{Author contributions}

Conceptualization: D.B., S.A.-S.; methodology: D.B., P.X., C.-P.H., D.Y., S.A.-S.; validation: D.B., S.A.-S.; formal analysis: D.B., P.X., S.A.-S.; investigation: D.B., P.X., D.Y., S.A.-S.; resources: C.D., N.M., H.N., V.L., W.H.; data curation: D.B., S.A.-S.; writing-original draft: D.B., S.A.-S.; writing-review and editing: D.B., P.X., V.L., C.-P.H. D.Y., S.A.-S.; visualization: D.B., S.A.-S.; supervision: W.H., D.Y., S.A.-S.; project administration: S.A.-S.; funding acquisition: D.Y., S.A.-S.

\section{Additional information}

Supplementary Information accompanies this paper at https://doi.org/10.1038/s41467019-12068-x.

Competing interests: The authors declare no competing interests.

Reprints and permission information is available online at http://npg.nature.com/ reprintsandpermissions/

Peer review information Nature Communications thanks the anonymous reviewers for their contribution to the peer review of this work.

Publisher's note Springer Nature remains neutral with regard to jurisdictional claims in published maps and institutional affiliations.

Open Access This article is licensed under a Creative Commons Attribution 4.0 International License, which permits use, sharing, adaptation, distribution and reproduction in any medium or format, as long as you give appropriate credit to the original author(s) and the source, provide a link to the Creative Commons license, and indicate if changes were made. The images or other third party material in this article are included in the article's Creative Commons license, unless indicated otherwise in a credit line to the material. If material is not included in the article's Creative Commons license and your intended use is not permitted by statutory regulation or exceeds the permitted use, you will need to obtain permission directly from the copyright holder. To view a copy of this license, visit http://creativecommons.org/ licenses/by/4.0\%

(C) The Author(s) 2019 